\title{
Incidence and etiology of maxillofacial trauma: a retrospective analysis of King Chulalongkorn Memorial Hospital in the past decade
}

\author{
Pornthep Pungrasmi*, Sahatad Haetanurak
}

\begin{abstract}
Background: Maxillofacial injury is a common injury in trauma patients. The incidence, associated injuries and causes have been never reported for King Chulalongkorn Memorial Hospital (KCMH).

Objectives: To report the incidence, associated injuries, age groups, treatments, and behavioral risks in maxillofacial patients who were admitted to KCMH in the past decade.

Methods: We conducted a retrospective descriptive analysis of patients from January 1, 2006, to December 31, 2015, to evaluate the age groups, causes, sites of facial bone fracture, associated injuries, treatments, and behavioral risks.

Results: There were 1,275 patients ( $79 \%$ male and $21 \%$ female). The most common age group was $21-30$ years (30.6\%), followed by $11-20$ years $(19.5 \%)$ and $31-40$ years (18.8\%). The most common cause of injury was motorcycle accident $(39.7 \%)$, and the most common associated injury was head injury $(58 \%)$. The total number of fractures were 1,526 , with the most common fracture site being the zygomaticomaxillary complex $(38.6 \%)$, followed by mandible $(21.8 \%)$ and nasal bone (17.8\%). Most fractures were treated using open reduction and internal fixation with plates and screws.

Conclusions: The main cause of maxillofacial injury is motorcycle accident even though the government launched a policy named "Decade of Action for Road Safety 2011-2020" to reduce road traffic deaths. Thailand continues to need stronger law enforcement to reduce risky motorcycle driving behavior.
\end{abstract}

Keywords: epidemiology, etiology, incidence, maxillofacial fracture, maxillofacial injury, Thailand

Maxillofacial fractures are common in trauma patients. The incidence of maxillofacial trauma varies from region to region globally. These fractures may be isolated or combined with other fractures and injuries including those in the head, spine, chest, abdomen, pelvis, and extremities. The epidemiology of maxillofacial fractures varies by age group, cause of the injuries, and type of fracture and also depends on the group studied. Moreover, such injuries are severe, resulting in complicated treatments and often unsatisfactory results [1]. Emergency clinicians and surgeons, who treat patients with maxillofacial fractures, can not only provide medical treatment but also play a role in reducing the problem as they can point out the importance of such problems to the public. Understanding the epidemiology of maxillofacial fractures

*Correspondece to: Pornthep Pungrasmi, Department of Plastic and Reconstructive Surgery, Faculty of Medicine, Chulalongkorn University, Bangkok 10330, Thailand, e-mail: pornthep.p@chula.ac.th Department of Plastic and Reconstructive Surgery, Faculty of Medicine, Chulalongkorn University, Bangkok 10330, Thailand (c) (1) ()๑ () 2017 Pornthep Pungrasmi, Sahatad Haetanurak

This Work is licensed under Creative Common License 
is important in establishing the treatment and prevention of these injuries.

In the present study, we determined the age groups, causes of injury, associated injuries, types of injuries, and methods of treatment for maxillofacial fractures at King Chulalongkorn Memorial Hospital (KCMH) in the past decade to help the health care providers in establishing policy to prevent these injuries and to aid the surgeons who are concerned about the type of maxillofacial injuries and the associated injuries in the treatment of these trauma patients. $\mathrm{KCMH}$ is a general and tertiary referral hospital, and is one of the largest hospitals in Thailand. The Plastic and Reconstructive Surgery Department is only one of the units that takes responsibility for maxillofacial injuries in $\mathrm{KCMH}$.

\section{Materials and methods}

All medical records and radiographs of patients admitted to $\mathrm{KCMH}$ because of maxillofacial fractures during the period from January1, 2006, to December 31, 2015, were reviewed. The information on distribution of age, sex, cause of injury, alcohol drinking, associated injuries, pattern, and treatment of maxillofacial fractures was collected and evaluated. We excluded medical records with unclear data and radiographs without a definite fracture site from the present study. Data were entered in Microsoft Excel, version 2010, and descriptive statistics including frequencies and percentages were calculated using IBM SPSS Statistics for Windows, version 22. This study was approved by the institutional review board (IRB) of the Faculty of Medicine, Chulalongkorn University, Bangkok, Thailand (IRB No. 357/59, Certificate of Approval No. 641/2016).

\section{Results}

In the decade from January 1, 2006, to December 31, 2015, 1,275 patients were admitted to $\mathrm{KCMH}$ because of maxillofacial fractures. Their mean age was 33.8 years (range 2-96 years). Male-to-female ratio was 3.7:1 as shown in Table 1. The incidence of maxillofacial fractures for each year is shown in Figure 1. The most common cause of maxillofacial fracture was motorcycle accident (39.7\%); other causes of maxillofacial fracture are presented in Table 2. Alcohol consumption was reported in $583(47.5 \%)$ cases, denied in $315(24.7 \%)$ cases, and in the remaining 377 (29.5\%) cases, it was not documented. Associated injuries, such as head injury, thoracic injury, abdominal injury, pelvic injury and cervical
Table 1. Sex and age distribution of patients with maxillofacial fracture

\begin{tabular}{lrrrrr}
\hline \multirow{2}{*}{ Age (years) } & \multicolumn{2}{c}{ Sex } & & \multicolumn{2}{c}{ Total } \\
\cline { 2 - 3 } \cline { 5 - 6 } & Male & Female & & Number & $\%$ \\
\hline $1-10$ & 14 & 6 & 20 & 1.6 \\
$11-20$ & 199 & 50 & 249 & 19.5 \\
$21-30$ & 324 & 66 & 390 & 30.6 \\
$31-40$ & 194 & 45 & 239 & 18.8 \\
$41-50$ & 161 & 34 & & 195 & 15.3 \\
$51-60$ & 64 & 33 & & 97 & 7.6 \\
$61-70$ & 24 & 12 & & 36 & 2.8 \\
$>70$ & 26 & 23 & & 49 & 3.8 \\
Total & 1006 & 269 & 1275 & 100 \\
\hline
\end{tabular}

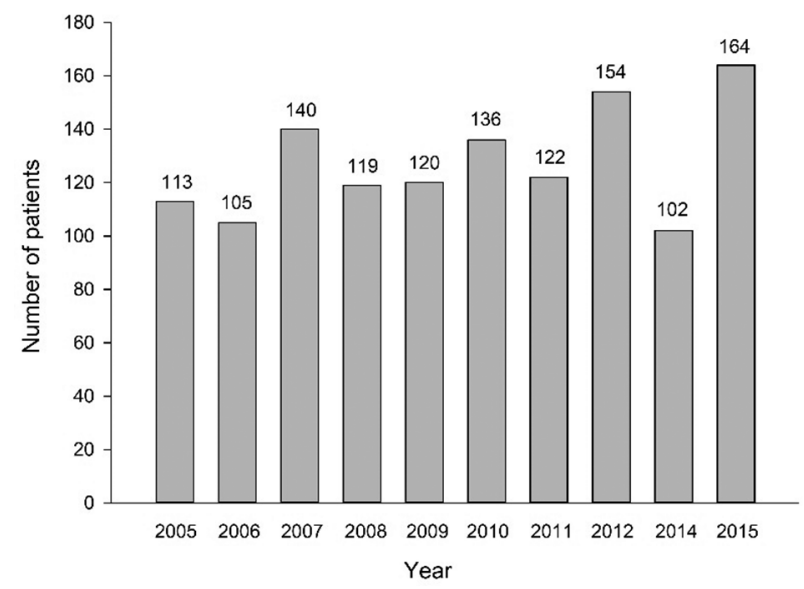

Figure 1. Incidence of maxillofacial fractures

Table 2. Causes of maxillofacial fractures

\begin{tabular}{lrrrrr}
\hline $\begin{array}{l}\text { Age } \\
\text { (years) }\end{array}$ & \multicolumn{5}{c}{ Causes } \\
\cline { 2 - 6 } & Motorcycle & Car & Falling & Assault & Others \\
\hline $1-10$ & 3 & 6 & 6 & 0 & 5 \\
$11-20$ & 134 & 10 & 17 & 62 & 26 \\
$21-30$ & 184 & 20 & 32 & 115 & 39 \\
$31-40$ & 79 & 15 & 30 & 75 & 40 \\
$41-50$ & 67 & 10 & 26 & 56 & 36 \\
$51-60$ & 22 & 13 & 20 & 23 & 19 \\
$61-70$ & 10 & 6 & 4 & 7 & 9 \\
$>70$ & 7 & 10 & 24 & 0 & 8 \\
Total & $506(39.7 \%)$ & $90(7.1 \%)$ & $159(12.5 \%)$ & $338(26.5 \%)$ & $182(14.2 \%)$ \\
\hline
\end{tabular}

(C-) spine injury, were found in 368 (28.9\%) cases, as shown in Figure 2.

The total number of fractures was 1,526 , which included zygomaticomaxillary complex (ZMC), mandible, nasal bone, maxilla, panfacial, and nasoorbitoethmoid complex, as given in Table 3. The specific types of mandibular fractures 


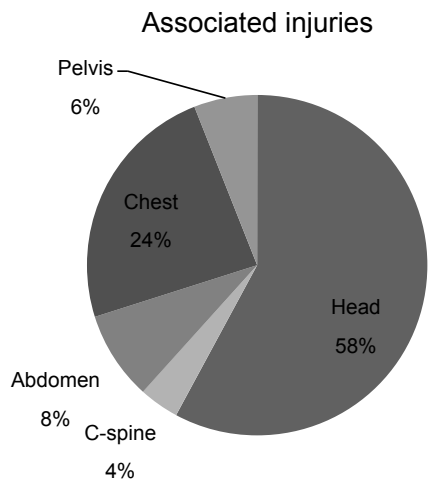

Figure 2. Associated injuries in patients with maxillofacial fracture

Table 3. Types of maxillofacial fracture

\begin{tabular}{lrrr}
\hline Fracture & \multicolumn{2}{c}{ Year } & Number \\
\cline { 2 - 3 } & $\mathbf{2 0 0 6 - 2 0 1 0}$ & $\mathbf{2 0 1 1 - 2 0 1 5}$ & \\
\hline ZMC & 264 & 325 & 589 \\
Maxilla & 46 & 84 & 130 \\
Nasal bone & 124 & 148 & 272 \\
Nasoorbitoethmoid & 17 & 35 & 52 \\
Mandible - total & 151 & 181 & 332 \\
Panfacial & 53 & 98 & 151 \\
\hline
\end{tabular}

ZMC, zygomaticomaxillary complex

Table 4. Specific types of mandibular fracture

\begin{tabular}{lr}
\hline Mandibular fractures & Number (\%) \\
\hline Single fracture & $\mathbf{2 0 4}(\mathbf{6 1 . 5 \% )}$ \\
Mandibular symphysis/parasymphysis & $85(25.6 \%)$ \\
Mandibular body only & $25(7.5 \%)$ \\
Mandibular angle only & $46(13.9 \%)$ \\
Mandibular condyle only & $48(14.5 \%)$ \\
Combined fracture $^{\dagger}$ & $\mathbf{1 2 8}(\mathbf{3 8 . 5 \% )}$ \\
Mandibular symphysis/parasymphysis + body & $13(3.9 \%)$ \\
Mandibular symphysis/parasymphysis + angle & $51(15.4 \%)$ \\
Mandibular symphysis/parasymphysis + condyle & $42(12.6 \%)$ \\
Mandibular body + angle & $5(1.50 \%)$ \\
Mandibular body + condyle & $7(2.1 \%)$ \\
Mandibular angle + condyle & $10(3.0 \%)$ \\
Total & $\mathbf{3 3 2 ( 1 0 0 \% )}$ \\
\hline
\end{tabular}

${ }^{+}$Combined fracture includes patients who have more than one fracture site

are listed in Table 4 and types of maxillary fractures are listed in Table 5. The treatments of fractures were open reduction and internal fixation, closed reduction, and conservative treatment. The details of such treatments are given in Table 6.
Table 5. Specific types of maxillary fracture

\begin{tabular}{lr}
\hline Le Fort fracture & Number (\%) \\
\hline Single fracture & $\mathbf{9 1 ( 7 0 \% )}$ \\
Le Fort I & $45(34.6 \%)$ \\
Le Fort II & $42(32.3 \%)$ \\
Le Fort III $_{\text {Combined fracture }}{ }^{+}$ & $4(3.1 \%)$ \\
Total $^{2}$ & $\mathbf{3 9 ( 3 0 \% )}$ \\
\hline
\end{tabular}

${ }^{+}$Combined fracture includes patients who have more than one fracture site

\section{Discussion}

Trauma patients need special care and treatment because of their associated injuries, which might affect the outcome of treatment and consequence of injuries. In the present study, ratio of men to women with maxillofacial trauma was 3.7:1, which is similar to the ratio found in other studies [2, 3]. These results show that men are at a higher risk of maxillofacial injuries than women. The most common age group was 21-30 years (390 [30.59\%]), followed by $11-20$ years (249 [19.5\%]), and $31-40$ years (239 [18.8\%]), which is consistent with the other studies [3-6] and in contrast with the study of Boonkasem et al. [2] that found that the number of patients in each age group was not different $(\sim 19 \%)$. However, the study by Boonkasem et al. included only 210 patients and was conducted in a rural area, whereas our study was conducted in a trauma center in an urban area.

\section{Cause of maxillofacial injuries}

The cause of maxillofacial injuries varies because of socioeconomic, cultural, and environmental factors. In our study, motorcycle accident (506 [40\%] cases) was the most common cause of injuries, which is similar to that seen in other studies [7, 8]. This may be a result of a societal shift from agricultural to industrial dependency, resulting in more traffic that might consequently cause more facial injuries from motorcycle accident. Apart from the low cost of motorcycles, Bangkok has some of the most severe traffic congestion in the world, encouraging the use of motorcycles in many situations. These data correlate with the World Health Organization's report that the leading cause of death in the age group of 15-29 years is road traffic accidents, mostly involving male victims, and the proportion is double in developing countries compared with developed countries.

By contrast, a multicenter and prospective study from the European Maxillofacial Trauma project in 2015 found 
Table 6. Treatment of patients with maxillofacial fracture

\begin{tabular}{lrrrrr}
\hline Fractures & \multicolumn{5}{c}{ Treatments } \\
\cline { 2 - 6 } & $\begin{array}{c}\text { Open reduction } \\
\text { and internal reduction } \\
\text { fixation with } \\
\text { plates and } \\
\text { screws }\end{array}$ & $\begin{array}{c}\text { Closed Conservative Others Total } \\
\text { Zygoma }\end{array}$ & & & \\
Mandible & 371 & 15 & 180 & 23 & 589 \\
Le Fort & 259 & 48 & 18 & 7 & 332 \\
Nasal bone & 108 & 9 & 5 & 8 & 130 \\
Nasoorbito- & - & 177 & 90 & 5 & 272 \\
ethmoid & 35 & 5 & 10 & 2 & 52 \\
Panfacial & 126 & 5 & - & 20 & 151 \\
\hline
\end{tabular}

that assault and falling are the most common causes of maxillofacial injuries with rates of $39 \%$ and $31 \%$, respectively. However, road traffic accidents cause maxillofacial injuries in only $11 \%$ of people [9]. The decrease in road traffic accidents in Europe is attributed mainly to the implementation of strictly enforced traffic rules and regulations with best practice on seat belts, drink driving, speed limits, motorcycle helmets, and child restraints [10].

In patients older than 70 years, falling is the most common cause of injury (24 of 49 [49\%] cases). Manodh et al. [11] and Al-Qamachi et al. [12] reported that $66.6 \%$ and $83 \%$ of maxillofacial trauma in elderly people, respectively, is a consequence of falls. The incidence of falling increases by age and mostly occurs at home because of senility, osteoporosis, and systemic complications such as diabetes.

\section{Associated injuries}

Associated injuries were found in $28.9 \%$ of patients with maxillofacial fracture (368 of 1,275 cases) and comprised head injury in $58 \%$ of patients, thoracic injury in $24 \%$, abdominal injury in $8 \%$, pelvic injury in $6 \%$, and $\mathrm{C}$-spine injury in 4\%, as shown in Figure 2, corresponding to the studies of Chalya et al. [3] and Gadre et al. [4] who found that head injuries occurred in $53.1 \%$ and $56 \%$ of patients with maxillofacial trauma, respectively. Head injuries are the most commonly associated injuries because the forces that can cause facial bone fractures might also cause brain injuries. Another study showed the most common associated injury was to the extremities (51.3\%), followed by skull injury (27.6\%) [13].

The most important associated injury is the $\mathrm{C}$-spine injury, which occurred in $4 \%$ of the patients. The incidence of $\mathrm{C}$-spine injuries varies from $0 \%$ to $8 \%$ in maxillofacial injuries. Mukherjee et al. [14] found that patients with mixed mandibular and nonmandibular fractures had a higher incidence of C-spine injuries than those with mandibular fracture only and nonmandibular fracture only, with the rates of $12.8 \%$, $1.8 \%$, and $1.5 \%$, respectively [14]. Vital transportation, e.g. by ambulance, and primary care of patients with maxillofacial injuries using $\mathrm{C}$-spine protection to avoid spinal cord injuries is necessary, especially in patients with combined mandibular and nonmandibular fractures who have a higher risk of C-spine injuries.

\section{Variation in maxillofacial injury}

The variation in the patterns of facial bone fracture depends on variation in geographic, cultural, and socioeconomic environments of the countries in which studies are conducted. In the present study, the most common facial fracture is ZMC fracture $(38.6 \%)$, followed by mandibular fracture $(21.8 \%)$ and nasal bone fracture $(17.8 \%)$. The direction of the injury-producing force determines the pattern of fractures, and the ZMC has the most prominent anatomical position in the Asian face $[15,16]$. Therefore, it is not surprising that the most common fracture in the present study is the ZMC fracture. The finding reported here is in contrast with that reported by Manodh et al. [11] who found that the most common maxillofacial fracture is mandibular fracture (59.2\%). They implied that the patients usually, as a reflex, turn to their side when there is a sudden impact directed to the face. Therefore, the mandible is the first bone that encounters the injury force, especially when the victim wears an open-type helmet. By contrast, the incidence of nasal bone fracture is quite low, compared with that in other studies $[17,18]$. It appears likely that in these other studies, the patients have more prominent nasal bones and lack soft tissue coverage, making the nasal bone the most fragile facial bone. By contrast, in the Asian faces in the present study, the malar bone is more prominent and the nasal dorsum is flatter $[15,16]$.

In the case of mandibular fractures, we found that 204 $(61.5 \%)$ cases had single fracture. Parasymphysis is the most common fracture site, as reported by Manodh et al. [11]. This may result in this area being anatomically the weakest point. Usually, the canine roots are longer than other roots, resulting in less bone and a higher tendency to fracture [17]. We found that $128(38.5 \%)$ cases had combined fracture, which is consistent with the study of Sirimaharaj and Pyungtanasup [5] and Park and Lim [6] who reported the incidence of combined fracture to be $41.4 \%$ and $36.7 \%$, respectively. The most common combined fracture was parasymphysis and angle (15.4\%), followed by parasymphysis and condyle (12.6\%), which is consistent with 
the report of Park and Lim [6], while the study from Parkland Memorial Hospital found that the most common combined fracture was parasymphysis and condyle (15\%), followed by parasymphysis and angle (8\%) [19]. This might be the result of different causes of injuries. The main cause of injury in the present study was road traffic accidents, which resulted in more impact force directed to the mandible. By contrast, the causes of injury in patients at Parkland Memorial Hospital were falling and assault. With these injuries, the impact force might be directed to the primary fracture site and then transmitted to the condyle, which is the thinnest part of mandible.

At $\mathrm{KCMH}$, Neurosurgery Unit is mainly responsible for frontal bone fracture and the Plastic Surgery Unit is responsible only for the anterior table of frontal sinus. We did not include frontal bone fracture in the present study because it was beyond our scope.

\section{Treatment of maxillofacial injury}

The main goal of treatment for facial fractures is to rearrange bone pieces in the same place (anatomical reduction) for good cosmetic results and function, especially occlusion [1]. Most patients therefore underwent open reduction and internal fixation with plates and screws $(58.9 \%)$. In cases where fractures occurred in both upper and lower jaws, patients underwent intermaxillary fixation for 1-3 weeks. However, patients with nasal fracture usually underwent either closed reduction or conservative treatment, owing to the small and slim Asian nasal bone, in which fracture might not be readily observed.

\section{Road safety}

In reference to the United Nation's policy of "improving global road safety" from the Moscow Declaration, urgent action is needed to achieve the ambitious target for road safety reflected in the newly adopted 2030 agenda for sustainable development, which will half the number of deaths and injuries from road traffic crashes globally by 2020 . The Thai government needs to enforce the laws with best practice on seat belts, drink driving, speed limits, motorcycle helmets, and child restraints. While there has been progress toward improving road safety legislation and in making vehicles safer, the present study shows that the pace of change is too slow. A study by Jiwattanakulpaisarn et al. [20] reported that only $60 \%$ of drivers and only $28 \%$ of passengers wore a motorcycle helmet. Nearly half of patients (583 patients, $(47.5 \%)$ reported having consumed alcohol, 315 patients $(24.7 \%)$ denied consumption, and approximately $30 \%$ did not indicate.
Surprisingly, after the policy launch, not only the incidence of maxillofacial injury increased by $14 \%$ (years 2006 2010, 597 cases vs years 2011-2015, 678 cases) but also the severity of injuries increased. The authors evaluated severity increase by comparing combined and multiple fractures with single fracture. We conclude that the Thai government needs to strictly enforce laws to promote risk reduction [20], and in further studies, we suggest that a prevention program and a supportive technology should be developed.

\section{Helmets and motorcycle injuries}

In addition to the force impact from the trauma, prevention of facial and maxillofacial injuries with helmets is very important to protect the victim from the direct force affecting the facial bones. Two types of helmets are used: closed and open. The closed type is available in a specific head size, but some motorcyclists use an improper size. As a result, protection might not be adequate. Some motorcyclists do not fasten the chin straps in the open type of helmets. If an accident occurs, the helmet cannot protect the facial bones effectively. Cooter and David [21] found that the use of a helmet significantly decreased neurotrauma in patients with facial fractures who wore an energy-absorbing helmet. Similarly, Harvey et al. [22] found that accident victims who did not wear helmets are more likely to sustain facial bone fractures and are more likely to require hospital admission than helmeted victims.

\section{Limitations}

Because this was a retrospective observational study, some data were not collected. For example, for patients who sustained maxillofacial fractures from motorcycle accidents, the use of helmets and alcohol consumption were not indicated in the records of approximately $30 \%$ of the cases. We recommend that for prospective studies such indicators need to be added to the medical record form and outpatient department and inpatient department record cards, so that the data can be analyzed and generalizations involving law enforcement can be made in order to reduce the risk factors in the long term. Because this was a single-center study, it may not represent the true situation in Bangkok.

\section{Conclusions}

The present study, the first report from $\mathrm{KCMH}$, which indicates the incidence, cause, severity, and methods of treatment 
for facial and maxillofacial injuries over the past decade, found that the incidence and severity of maxillofacial injuries increased, even though the government launched a policy named "Decade of Action for Road Safety 2011-2020" to reduce road traffic deaths. The main cause of maxillofacial injury is motorcycle accident. We conclude that Thailand needs stronger law enforcement to reduce risky motorcycle driving behavior.

Author contributions. PP and SH made substantial contributions to the conception and design, acquisition, analysis, and interpretation of data in the present study. SH drafted the manuscript, and PP critically revised it. Both authors approved the final version submitted for publication and take responsibility for the statements therein.

Acknowledgments. The authors thank Miss Kalaya Khongkhaprasert and Miss Pornprapa Fahpinyo, Department of Medical Records and Statistics KCMH, for searching records and data collection.

Conflict of interest statement. All authors have completed and submitted the ICMJE form for disclosure of potential conflicts of interest. The authors disclose no conflict of interest.

\section{References}

[1] Ricketts S, Gill HS, Fialkov JA, Matic DB, Antonyshyn OM. Facial fractures. Plast Reconstr Surg. 2016; 137:424e-44e.

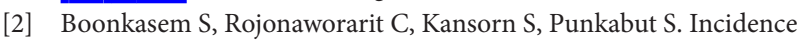
and etiology of maxillofacial trauma: a retrospective analysis of patients attending a provincial hospital in northern Thailand. J Pub Health Dev. 2015; 13:57-71.

[3] Chalya PL, McHembe M, Mabula JB, Kanumba ES, Gilyoma JM. Etiological spectrum, injury characteristics and treatment outcome of maxillofacial injuries in a Tanzanian teaching hospital. J Trauma Manag Outcomes. 2011; 5:7. doi:10.1186/1752-2897-5-7.

[4] Gadre KS, Halli R, Joshi S, Ramanojam S, Gadre PK, Kunchur R, et al. Incidence and pattern of cranio-maxillofacial injuries: a 22 year retrospective analysis of cases operated at major trauma hospitals/ centres in Pune, India. J Maxillofac Oral Surg. 2013; 12:372-8.

[5] Sirimaharaj W, Pyungtanasup K. The epidemiology of mandibular fractures treated at Chiang Mai University Hospital: a review of 198 cases. J Med Assoc Thai. 2008; 91:868-74.

[6] Park KP, Lim SU. Fracture patterns in the maxillofacial region: a four-year retrospective study. 2015; 41:306-16.
[7] Zix JA, Schaller B, Lieger O, Saulacic N, Thorén H, Iizuka T. Incidence, aetiology and pattern of mandibular fractures in central Switzerland. Swiss Med Wkly. 2011; 141:w13207. doi:10.4414/ smw.2011.13207.

[8] Reilly PL, David DJ. Craniofacial injuries. In: Winn HR, editor. Youmans and Winn neurological surgery. vol. 4. 7th ed. New York: Elsevier; 2016, p. 2952-70.

[9] Boffano P, Roccia F, Zavattero E, Dediol E, Uglesic V, Kovacic Z, et al. European Maxillofacial Trauma (EURMAT) project: a multicentre and prospective study. J Craniomaxillofac Surg. 2015; 43:62-70.

[10] Hallmer F, Anderud J, Sunzel B, Guner N, Andersson G. Jaw fractures diagnosed and treated at Malmo University Hospital: a comparison of three decades. Int J Oral Maxillofac Surg. 2010; 39:446-51.

[11] Manodh P, Prabhu Shankar D, Pradeep D, Santhosh R, Murugan A. Incidence and patterns of maxillofacial trauma - a retrospective analysis of 3611 patients - an update. Oral Maxillofac Surg. 2016; 20:377-83.

[12] Al-Qamachi LH, Laverick S, Jones DC. A clinico-demographic analysis of maxillofacial trauma in the elderly. Gerodontology. 2012; 29:e147-9.

[13] Zhou HH, Liu Q, Yang RT, Li Z, Li ZB. Maxillofacial fractures in women and men: a 10-year retrospective study. J Oral Maxillofac Surg. 2015; 73:2181-8.

[14] Mukherjee S, Abhinav K, Revington PJ. A review of cervical spine injury associated with maxillofacial trauma at a UK tertiary referral centre. Ann R Coll Surg Engl. 2015; 97:66-72.

[15] Mahatumarat C, Rojvachiranonda N. Reduction malarplasty without external incision: a simple technique. Aesthetic Plast Surg. 2003; 27:167-71.

[16] Wen YF, Wong HM, Lin R, Yin G, McGrath C. Inter-ethnic/racial facial variations: a systematic review and Bayesian meta-analysis of photogrammetric studies. PLoS One. 2015; 10:e0134525. doi:10.1371/ journal.pone.0134525.

[17] Lee JH, Cho BK, Park WJ. A 4-year retrospective study of facial fractures on Jeju, Korea. J Craniomaxillofac Surg. 2010; 38:192-6.

[18] Hyman DA, Saha S, Nayar HS, Doyle JF, Agarwal SK, Chaiet SR. Patterns of facial fractures and protective device use in motor vehicle collisions from 2007 to 2012. JAMA Facial Plast Surg. 2016; 18:455-61.

[19] Morris C, Bebeau NP, Brockhoff H, Tandon R, Tiwana P. Mandibular fractures: an analysis of the epidemiology and patterns of injury in 4,143 fractures. J Oral Maxillofac Surg. 2015; 73:951.el-e12.

[20] Jiwattanakulpaisarn P, Kanitpong K, Ponboon S, Boontob N, Aniwattakulchai P, Samranjit S. Does law enforcement awareness affect motorcycle helmet use? Evidence from urban cities in Thailand. Glob Health Promot. 2013; 20:14-24.

[21] Cooter RD, David DJ. Motorcyclist craniofacial injury patterns. [online] 2013 [cited 2017 Aug 16]. Available from: http://www. bikersrights.com/statistics/cooter/cooter.html

[22] Harvey JA, Gibreel W, Charafeddine A, Sharaf B. Helmet wear and craniofacial trauma burden: a plea for regulations mandating protective helmet wear. Craniomaxillofac Trauma Reconstr. 2017; 10:197-203. 